\title{
Companding technique for high dynamic range measurements using Gafchromic films
}

\author{
Frank Van den Heuvel, Wouter Crijns, and Gilles Defraene \\ Department of Experimental Radiotherapy, \\ Catholic University of Leuven, \\ Leuven, Belgium
}

Purpose: To introduce a methodology to perform dose measurements using Gafchromic films which can span several decades of dose levels.

Methods: The technique is based on a rescaling approach using different films irradiated at different dose levels. This is combined with a registration protocol correcting positioning and scaling factors for each film.

The methodology is validated using TLD's for out-of-field doses. Furthermore, two examples are provided using the technique to characterize small sized radiosurgery cones and compared to measurements made with a pinpoint chamber.

Results: Excellent agreement with TLD, planning systems and measurement was found. The superior resolution of the film technique was apparent.

Conclusions: We have introduced a new technique allowing users to quantify very low doses in conjunction with commissioning measurements. The use of film also provides $2 \mathrm{D}$ information on beam characteristics in high resolution measurements.

\section{INTRODUCTION}

The use of radiochromic films as a measurement device has become popular during the last few years. There are many reasons for this among which: 1) high resolution, 2) no additional development needed, 3) off the shelf reading tools, and 4) tissue equivalence. Some disadvantages include: 1) variations in sensitivity 2) heterogeneity within a film does exist, and 3) a limited dynamic range does not allow the films to be used to quantify beam characteristics or to measure relatively low doses. Of these disadvantages the last is the most important as batch production and pre-scanning allow users to minimize the first two problems.

The effect of a limited dynamic range of Gafchromic film is to generate noise at high dose levels as well as very low dose levels. In both cases the source of noise is due to quantum noise. In the case of the low dose, the sensitivity of the film combined with a low number of interactions with the film provide a noisy signal when read. On the other end of the dose spectrum the film saturates and measuring the optical transmittance of the film again produces quantum noise.

In this paper we describe a technique to extend the dynamic range to low doses by making use of a rescaling algorithm inspired by companding noise reduction in electrical or audio signals. Indeed the variation of dose can be considered as a function of 2 dimensions on the film which has been altered by the response of the Gafchromic film. This response function is not linear and has two stages. Firstly, the response of the film to the dose which alters the transmittance of the film. Secondly, the readout stage which is again a measuring device with a non-linear response. The first stage will introduce noise in the low dose region due to counting statistics. The second stage introduces noise again through counting statistics, but this time due to the paucity of optical photons that can be detected in regions of high opacity.

Companded transmission is a technique that has been used to transmit data over noisy telegraph lines ${ }^{1}$ and has also been used in the audio industry to reduce cassette tape noise ${ }^{2}$ as well as employed to more efficiently digitize sound tracks ${ }^{3}$. In essence a signal or part of a signal is changed before transmission to fit in the optimal bandwidth of the transmitter after which it is restored to its normal level before being fed to the output circuit. More descriptively, any part of the signal which would be overly distorted by the transmission procedure is scaled (or compressed) to fit within the optimal response range of the transfer function. After transmission a rescaling (or expansion) takes place to reconstruct the original signal. The word companding is a combination of compression and expanding.

In order to apply this concept to film measurements, which is not a dynamic environment, we take different films at different levels of dose. This means that we deliver a different number of monitor units (MU) to the films, each dose level bringing another part of the dose profile within the optimal range (compression). After digitization of the film, each pixel falling within that dynamic range is selected while the others are discarded. The pixel values are then rescaled to the original reference dose (expansion). The information of all films irradiated at different levels is then recombined. Within the analogy of signal transmission we transmit the signal several times using different amplification levels and recombine everything after reception and expansion.

In addition, we will show that this approach allows users to perform small field measurements with superior resolution than heretofore possible. 


\section{METHODS AND MATERIALS}

\section{A. Films}

We use EBT2 Gafchromic films provided by International Specialty Products ${ }^{4}$. The films are scanned at a resolution of 150 dpi using a 10000XL Epson scanner. The films are batch calibrated to provide absolute dose values. Two films represent the complete batch and is calibrated using a two film methodology. We chose to use transmittance expressed as a function of dose using the following expression:

$$
T=T_{\infty}+\frac{T_{0}-T_{\infty}}{1+\frac{D}{\beta}}
$$

where $T_{\infty}$ is the transmittance after delivering an infinite dose (or maximal opacity) and $T_{0}$ is the transmittance of a blank film. The parameter $\beta$ is a scaling factor for the dose $D$. This procedure has been described elsewhere and is available online (http://arxiv.org/abs/1107.3780) ${ }^{5}$. It provides an absolute error level of the order of 3 to $5 \%$ using the red channel output. The films are calibrated to provide accurate dosimetry between 0.2 Gy and 4 Gy, exhibiting limited noise. Note, that the calibration technique is not essential, any well calibrated film set can be used. Such techniques have been described by Micke et al. ${ }^{6}$. More general techniques for different types of film including relating optical density to dose using exponential $^{7-9}$ and polynomial ${ }^{10,11}$ calibration functions.

\section{B. Companding technique}

Let $L$ and $U$ be the lower and upper limits, respectively, of the calibration defining the limits within which the response of the film is deemed accurate. For the first film or reference film a maximal dose $D_{0}$ is delivered which is lower than $U$ but close to it. If the experiment calls for a dose which does not meet these criteria a multiplication factor $F_{0}$ can be chosen to make it so. Subsequent films are irradiated with a maximal dose $D_{i}$ that is a multiple of each previous film $D_{i}=D_{i-1} F_{i}$, with the emphasis factor $F_{i}$ such that $1<F_{i}<U / L$ for all $i>0$, guaranteeing overlap between subsequent films.

All films are converted to dose using a calibration protocol that links the transmittance to dose.

\section{Registration}

As the films exhibit a high resolution $(1$ pixel $\simeq 0.16$ $\mathrm{mm}$ ) it is difficult to exactly reproduce the position of a given film. Additionally, variations in the beam output of a linear accelerator introduces an uncertainty in the dose delivered and thus in the factor used to de-emphasize the signal. Finally, variations in film sensitivity within a single batch. To solve this we introduced an alignment protocol taking into account in both dosimetric and spatial variations. This is not a straightforward problem as the geometric registration and the dose level are not independent parameters.

The registration between two films of subsequent level of companding is performed as follows:

- We define the registration as a 4-dimensional vector $\left(X_{i}, Y_{i}, \theta_{i}, f_{i}\right)$, from film $i$ to film $i-1$ with $X_{i}$ and $Y_{i}$ being corrections on the translational coordinates and $\theta_{i}$ a rotational parameter; the component $f_{i}$ denotes the correction of the emphasis factor.

- The films are converted to dose using the aforementioned calibration. Only data that falls within the upper limit $(U)$ and lower limit $(L)$ of calibration is retained (see below).

- The two films are registered using data that is contained in the overlap between the two calibration ranges. This is dependent on the position of the film as well as the dose factor.

- The cost function to register the images is the dose difference between the two images multiplied by the gradient image $G=1+a \times \sqrt{G_{X}^{2}+G_{Y}^{2}}$, with $G_{X}$ and $G_{Y}$ the gradient calculated in the $\mathrm{X}$ and $\mathrm{Y}$ direction of the film which are chosen to follow the film edges. The parameter $a$ is set at 500 to allow the gradient function to have an impact on the images used as the gradient image is normalized to have an integrated amplitude of 1 .

- The cost function is optimized using a constrained minimization algorithm as implemented in the function fmincon in Matlab $^{12}$, with the option interior-point approach.

- The minimization procedure is performed in a progressive manner starting from a sub-sampled image at $50 \mathrm{dpi}$, then $75 \mathrm{dpi}$ and finally a full resolution image at $150 \mathrm{dpi}$. For every increase in resolution the constraints within which the optimization is performed are tightened. They are respectively $\left(5,5,10^{\circ}, 10 \%\right),\left(0.1,0.1,0.5^{\circ}, 0.5 \%\right)$, and $\left(0.03,0.03,0.05^{\circ}, 0.15 \%\right)$, using the same notation as for the transformation vector with all spatial values in centimeters.

\section{Combining the data}

Let $n$ be the number of films used; the value of the measurement at position $(x, y)$ of the $i$-th film is denoted by $M_{i}(x, y)$. The coordinates $(x, y)$ are registered and denote the position in the reference film. The multiplication factors are also denoted as outlined before, being $F_{i}$ indexed per film $i$ and corrected for variations during the registration procedure. Then the 
actual dose readout on this film is denoted as $R_{i}(x, y)$, and is calculated as follows:

$$
R_{i}(x, y)= \begin{cases}\frac{M_{i}(x, y)}{\prod_{j=0}^{i} F_{j}} & \text { if } M_{i}(x, y) \in[L, U] \\ 0 & \text { if } M_{i}(x, y) \notin[L, U]\end{cases}
$$

Finally, the resulting data of all $n$ films are summed to provide a combined data set. Pixels having a nonzero value in more than one film are averaged over the films. Mathematically this expressed as:

$$
\mathbf{R}(x, y)=\sum_{i=0}^{n} \frac{R_{i}(x, y)}{N(x, y)}
$$

Where $N(x, y)$ denotes the number of times a nonzero pixel is found at the position $(x, y)$ for all films. If $N(x, y)$ equals zero then the term $\frac{R_{i}(x, y)}{N(x, y)}=0$

The end result is a dose image of high resolution and dynamic range that can be used to characterize radiation beams in treatment planning systems or to provide data on radiation dose levels outside of the field edge. An example of a resulting data set is provided in Figure 1.

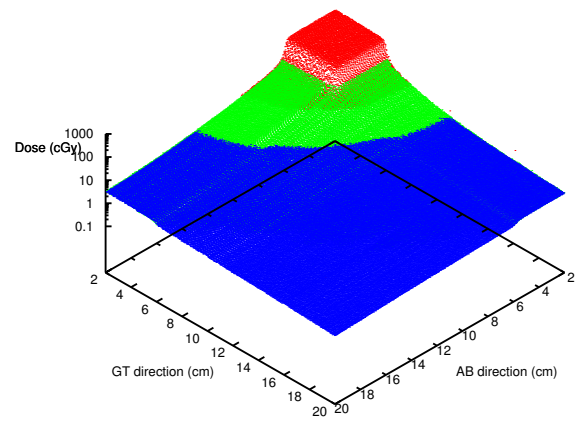

FIG. 1. Combination of three decades of irradiation data for a $10 \times 10 \mathrm{~cm}^{2}$ sized beam. The z-axis is logarithmic and in cGy, the other axes are in $\mathrm{cm}$

\section{Recipe}

For people wanting to use this type of measurement we provide a step-by-step approach:

1. Determine the maximum dose that will be delivered to your film in your measurement by other means or calculation.

2. Change the output of the machine (i.e. number of Monitor Units (MU)) so that this maximum falls within the optimal range of the film, preferably towards the upper end. This defines $F_{0}$.

3. Irradiate the film and note the geometry.
4. A second film is irradiated in the same conditions, using a higher number of MU. Choose the MU to reflect the multiplication factor $F_{i}$ as defined above.

5. Repeat this for as many films as are needed to reach the desired dynamic range.

6. Convert each film to a dose image.

7. Register the dose images geometrically and dosimetrically as outlined above.

8. Calculate the readout $R_{i}(x, y)$ for each film as in equation 2 .

9. Combine all data to generate an overall measurement using the expression in equation 3 .

\section{Validation}

The validation is performed with a single set-up. A stack of solid water is irradiated using a clinical $6 \mathrm{MV}$ photon beam from a Varian C/D 2100 linear accelerator. We present three different tests.

1. A $10 \times 10 \mathrm{~cm}^{2}$ field is compared to the results from a planning system (Varian Eclipse ${ }^{\mathrm{TM}}$ ) with the pencil beam and the AAA-algorithms. The Gafchromic films are placed at a depth of $10 \mathrm{~cm}$, with the surface of the stack located at $90 \mathrm{~cm}$ from the source. To obtain the measurement three films where used using an emphasis factor $F=10$, which was also used in all other examples.

In addition the low dose area is compared to measurements using TLD's. This data set has also been used in the ALLEGRO project ${ }^{14,15}$ to compare with TPS data. Here we add the TLD validation.

2. A Brainlab ${ }^{\mathrm{TM}}$ radiosurgery cone with an opening size of $30 \mathrm{~mm}$ and the machine jaws set to a $5 \times 5 \mathrm{~cm}^{2}$ size is compared to the data obtained for entry in the Brainscan ${ }^{\mathrm{TM}}$ planning system. For this purpose the field is measured with a pinpoint chamber $^{13}$ type 31006 of size $0.015 \mathrm{~cm}^{3}$ using a water phantom. The Gafchromic films are placed at a depth of $7.5 \mathrm{~cm}$, with the surface of the stack located at $92.5 \mathrm{~cm}$ from the source.

3. In an identical setup a cone with a diameter of $7.5 \mathrm{~mm}$ was also assessed.

\section{TLD}

LiF:Mg,Ti TLD's (TLD-100 EXTRAD, Harshaw) are used. Calibration of the dosimeters was performed in a 6 MV beam at $5 \mathrm{~cm}$ depth in a polystyrene phantom. The TLD's are read-out in a Harshaw 6600 hot gas reader, 
equipped with a ${ }^{90} \mathrm{Y} /{ }^{90} \mathrm{Sr}$ source. In order to minimize initial fading effects, read-out of calibration TLD's that have been irradiated with the internal source simultaneously with the experiment, is part of the standard processing procedure.

\section{RESULTS}

\section{A. $10 \times 10 \mathrm{~cm}^{2}$ fields}

Figure 2 provides a graph for the open field test. It is clear that the TLD's agree well with the film measurements. With doses to TLD's of this magnitude the contribution of the background can cause an offset in the measurements. In this case we did not correct for the offset which would explain the slight increase of the reading with respect to the Gafchromic measurement. Care was taken however to anneal the TLD's shortly before use. A good agreement with the planning system is found when AAA is used. In contrast, the pencil beam data does not agree well.

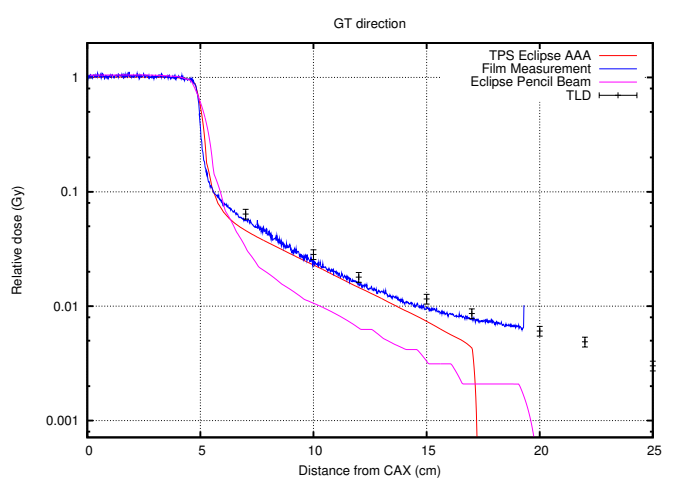

FIG. 2. Validation of a classic radiation therapy beam with a $6 \mathrm{MV}$ energy. The AAA implementation follows the measurement excellently. Moreover the TLD measurements show good agreement. A slight offset can be expected due to some background dose built up in the TLD's. Also note the difference in the field edge between planning and film measurement. The TPS data was modelled using data obtained with a compact IC 10 ion chamber (Wellhöfer).

\section{B. Small-field dosimetry $30 \mathrm{~mm}$ cone}

Figure 3 shows a log plot of the relative dose for a 30 $\mathrm{mm}$ cone. As in the classical field one can notice the difference in resolution of the Gafchromic film compared to the measurement. Although a pinpoint chamber was used to perform this measurement, a broadening of the field edge compared to the film is easily discernible.

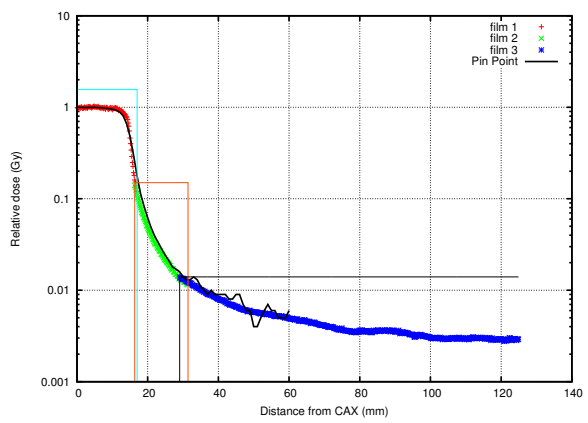

FIG. 3. A $30 \mathrm{~mm}$ cone delivers $6 \mathrm{MV}$ photons on the films.We compare to the results from a pinpoint chamber. The blue, orange and black squares delineate the spatial and dosimetric limitations for respectively the first, second, and third decade. Note that even while the pin-point chamber is small a difference can be seen in the resolving power compared to film measurements.

\section{Small-field dosimetry $7.5 \mathrm{~mm}$ cone}

In the case of the data obtained with irradiation using the smallest cone, as shown in Figure 4 there is no plateau in the data measured using the pinpoint chamber, leading us to believe that there are partial volume effects in play already. The film measurement does show a plateau phase. Also the ratio of the maximal measured dose compared to the output from a 10x10 field (output ratio) was more than double with film than with the pinpoint chamber. Due to these differences we have never used the smallest cone clinically. Furthermore, away from the central axis we note an increase in dose from $0.2 \%$ to $0.5 \%$.

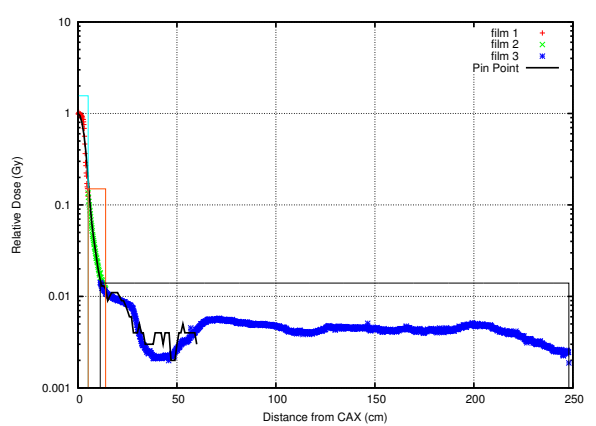

FIG. 4. A $7.5 \mathrm{~mm}$ diameter cone delivers $6 \mathrm{MV}$ photons on the films.We compare to the results from a pinpoint chamber. The blue, orange and black squares delineate the spatial and dosimetric limitations for respectively the first, second, and third decade. Note that even while the pin-point chamber is small a difference can be seen in the resolving power compared to film measurements. Also note that further away from the central axis in the region not measured by the ion chamber the dose increases and more than doubles. 


\section{Alignment}

In table I the transformation needed to match the different films show that there was a difference in machine output or response of the films used to measure the lower dose compared to the first film. It also shows that reasonable accuracy can be reached by mechanically positioning the films correctly in treatment as well as readout position. However, although shifts between consecutive films are of the order of less than $0.5 \mathrm{~mm}$, in the case of very steep gradients important inconsistencies can be introduced when using manual alignment only. The latter becomes important in the case of very small fields.

\begin{tabular}{|l|c|c|}
\hline $\mathbf{1 0} \times \mathbf{1 0}$ Field & Decade 1-2 & Decade 1-3 \\
\hline X-shift & $0.65 \mathrm{~mm}$ & $1.99 \mathrm{~mm}$ \\
\hline Y-shift & $0.64 \mathrm{~mm}$ & $3.35 \mathrm{~mm}$ \\
\hline Rotation & $-0.89^{\circ}$ & $-1.29^{\circ}$ \\
\hline Correction for $F_{i}\left(f_{i}\right)$ & 1.037 & 1.071 \\
\hline Cone $\mathbf{3 0} \mathbf{~ m m}$ & Decade 1-2 & Decade $1-3$ \\
\hline X-shift & $-0.00 \mathrm{~mm}$ & $-0.24 \mathrm{~mm}$ \\
\hline Y-shift & $-0.15 \mathrm{~mm}$ & $0.36 \mathrm{~mm}$ \\
\hline Rotation & $-0.04^{\circ}$ & $-0.33^{\circ}$ \\
\hline Correction for $F_{i}\left(f_{i}\right)$ & 0.934 & 0.957 \\
\hline \hline Cone 7.5mm & Decade 1-2 & Decade 1-3 \\
\hline X-shift & $0.58 \mathrm{~mm}$ & $-0.45 \mathrm{~mm}$ \\
\hline Y-shift & $0.07 \mathrm{~mm}$ & $1.53 \mathrm{~mm}$ \\
\hline Rotation & $-2.99^{\circ}$ & $-2.76^{\circ}$ \\
\hline Correction for $F_{i}\left(f_{i}\right)$ & 1.08 & 1.17 \\
\hline
\end{tabular}

TABLE I. Transformations performed to align the different films in this setup. The third film is matched to the second film. The data given are for all films with respect to the first film. This implies that errors for the third film are cumulative.

\section{DISCUSSION}

The approach as outlined here has been used to perform measurements within the framework of the ALLEGRO project ${ }^{14,15}$. The goal of one of the workgroups was to determine the dose outside of the high dose volume, in order to estimate the impact of newer radiotherapy techniques on the whole body dose. A second question to answer was whether data from commercial planning systems would be able to serve as a resource to obtain information on low-dose exposures. It was therefore necessary to enhance the accuracy of low dose measurements.

From the work in this paper and the comparisons with TLD measurements and ion-chamber data, we conclude that this technique provides data that not only allows estimates of low-dose contributions to patients, but also provides spatial information. The use of Gafchromic EBT2 film in this fashion will also allow its use in the characterization of radiation systems that deliver doses to very small volumes.

The registration algorithm is a necessary step in this approach. From the data shown here it becomes clear that there are many variables in the alignment of the films, including positioning during the dose delivery and during film readout. Such inaccuracies are cumulative when using more than two films as can be seen from the registration results which require larger shifts between decades 3 to 1 compared to decades 2 to 1 .

Finally, the choice of registration algorithm was done in an ad-hoc fashion as we wanted to provide proof of concept. Registration took the order of 30 minutes using an off the shelf modern computer. It is clear that there is progress to be made by optimizing the code and/or choice of registration algorithm.

\section{ACKNOWLEDGEMENTS}

This study was partly funded by the ALLEGROproject (7th Euratom Framework Programme for Nuclear Research and Training, Fission-2008-3.2.1).

\section{AUTHOR CONTRIBUTIONS}

Mr. Van den Heuvel outlined the idea and approach and provided a proof of concept. Mr Crijns actualized the registration component. Mr Defraene provided the TLD validation and tested the registration algorithm. All authors contributed to discussions and writing the article.

${ }^{1}$ A.B. Clark. Electrical picture-transmitting system. United States patent, 1691147, 1928.

${ }^{2}$ Ray Dolby. An audio noise reduction system. J. Audio Eng. Soc, 15(4):383-388, 1967.

${ }^{3}$ Steven W. Smith. Audio processing. In The scientist and engineer's guide to digital signal processing. California Technical Publishing, San Diego, CA, USA, 1997.

${ }^{4}$ ISP, New Jersey, USA.

${ }^{5}$ W. Crijns, , F. Maes, and F. Van den Heuvel. Gafchromic film dosimetry: calibration methodology and error analysis. Submitted to Med Phys, 2011.

${ }^{6}$ Andre Micke, David F. Lewis, and Xiang Yu. Multichannel film dosimetry with nonuniformity correction. Medical Physics, 38(5):2523-2534, 2011.

${ }^{7}$ L. J. van Battum and H. Huizenga. The curvature of sensitometric curves for Kodak XV-2 film irradiated with photon and electron beams. Med Phys, 33:2396-2403, Jul 2006.

${ }^{8}$ X. R. Zhu, S. Yoo, P. A. Jursinic, D. F. Grimm, F. Lopez, J. J. Rownd, and M. T. Gillin. Characteristics of sensitometric curves of radiographic films. Med Phys, 30:912-919, May 2003.

${ }^{9}$ J. F. Williamson, F.M. Khan, and S. C. Sharma. Film dosimetry of megavoltage photon beam: A practical method of isodensityto-isodose curve conversion. Med. Phys., 8:94-98, Jan./Feb. 1981.

${ }^{10}$ N. L. Childress, L. Dong, and I. I. Rosen. Rapid radiographic film calibration for IMRT verification using automated MLC fields. Med Phys, 29:2384-2390, Oct 2002.

${ }^{11}$ D. Georg, B. Kroupa, P. Winkler, and R. Potter. Normalized sensitometric curves for the verification of hybrid IMRT treatment plans with multiple energies. Med Phys, 30:1142-1150, Jun 2003. 
${ }^{12} \mathrm{~T}$. F. Coleman and Y. Li. On the convergence of interior-reflective Newton methods for nonlinear minimization subject to bounds. Mathematical Programming, 67(1):189-224, 1994.

${ }^{13}$ PTW Freiburg, Germany.

${ }^{14}$ G. Defraene and F. Van den Heuvel. Peripheral doses in prostate cancer radiotherapy: A comparison between IMRT and RapidArc treatments. Radiotherapy and Oncology, 99(Supplement
1):S127 - S127, 2011

${ }^{15}$ M. Durante, L. Sihver, E. Aird, B.A. Jereczek-Fossa, and F. Van den Heuvel. Dosimetry for normal tissue risk assessment: Measurement and calculation of the doses received by normal tissues from radiation therapy from current and emerging modalities. Radiotherapy and Oncology, 99(Supplement 1):S46 - S46, 2011. 